\title{
The Effects of the Vestibular Rehabilitation on the Benign Paroxysmal Positional Vertigo Recurrence Rate in Patients with Otolith Dysfunction
}

\author{
Reza Hoseinabadi ${ }^{1}$, Akram Pourbakht ${ }^{2}$, Nasrin Yazdani ${ }^{3}$, Ali Kouhi ${ }^{3}$, Mohammad Kamali ${ }^{4}$, \\ Farzaneh Zamiri Abdollahi ${ }^{1}$, and Sadegh Jafarzadeh ${ }^{5}$ \\ ${ }^{1}$ Department of Audiology, School of Rehabilitation, Tehran University of Medical Sciences, Tehran, Iran \\ ${ }^{2}$ Department of Audiology, Rehabilitation Research Centre, School of Rehabilitation Sciences, Iran University of Medical Sciences, \\ Tehran, Iran \\ ${ }^{3}$ Otorhinolaryngology Research Center, Amir-Alam Hospital, Tehran University of Medical Sciences, Tehran, Iran \\ ${ }^{4}$ Department of Rehabilitation Management, School of Rehabilitation Sciences, Iran University of Medical Sciences, Tehran, Iran \\ ${ }^{5}$ Department of Audiology, School of Paramedical Sciences, Mashhad University of Medical Sciences, Mashhad, Iran
}

\begin{abstract}
Received February 16, 2018
Revised April 17,2018

Accepted April 27, 2018
\end{abstract}

Background and Objectives: Although repositioning maneuvers have shown remarkable success rate in treatments of benign paroxysmal positional vertigo (BPPV), the high recurrence rate of BPPV has been an important issue. The aims of present study were to examine the effects of otolith dysfunction on BPPV recurrence rate and to describe the effect of vestibular rehabilitation exercises on BPPV recurrence in BPPV patients with concomitant otolith dysfunction. Subjects and Methods: Forty-five BPPV patients included in this study (three groups) Patients in group 1 had no otolith dysfunction and patients in groups 2 and 3 had concomitant otolith dysfunction. Otolith dysfunction was determined with ocular/cervical vestibular evoked myogenic potential (oVEMP and cVEMP) abnormalities. Epley's maneuver was performed for the patients in all groups but patients in group 3 also received a 2-month vestibular rehabilitation program (habituation and otolith exercises). Results: This study showed that BPPV recurrent rate was significantly higher in patients with otolith dysfunction in comparison to the group $1(p<0.05)$. Vestibular rehabilitation resulted in BPPV recurrence rate reduction. Utricular dysfunction showed significant correlation with BPPV recurrence rate. Conclusions: Otolith dysfunction can increase BPPV recurrence rate. Utricular dysfunction in comparison to saccular dysfunction leads to more BPPV recurrence rate. Vestibular rehabilitation program including habituation and otolith exercises may reduce the chance of BPPV recurrence.

J Audiol Otol 2018;22(4):204-208
KEY WORDS: Otolith dysfunction · Vestibular rehabilitation .

Benign paroxysmal positional vertigo.

\section{Introduction}

Benign paroxysmal positional vertigo (BPPV) is the most common vestibular disorder that causes true vertigo [1]. It occurs spontaneously in many patients, so identification of the underlying cause may always not be possible [2]. The

This is an Open Access article distributed under the terms of the Creative Commons Attribution Non-Commercial License (https://creativecommons.org/licenses/by-nc/4.0/) which permits unrestricted non-commercial use, distribution, and reproduction in any medium, provided the original work is properly cited. most common causes of BPPV include head trauma, labyrinthitis, and anterior vestibular artery ischemia, prolonged bed rest [3] and vestibular neuritis [4]. It is characterized by brief episodes of true vertigo induced by changing the head position in the plane of the involved semicircular canals. The diagnosis is confirmed by a positioning test called Dix-Hallpike maneuver which reveals vertigo and/or nystagmus. There are two forms of BPPV including canalithiasis and cupulolithiasis [2]. BPPV most commonly affects the posterior canal. However, it may affect any of the semicircular canals and any 
combinations of the multiple canals simultaneously [5].

The displacement of the otoconia particles from utricle to the semicircular canal is the most convincing theory to explain BPPV symptoms [6]. This theory is a basic concept for BPPV treatment approaches [7]. Canalith repositioning maneuver (CRM) or Epley's maneuver is the most commonly used approach and in 70-100\% of instances is successful [8]. The effectiveness of Epley's maneuver as the most successfully used maneuver in the treatment of posterior canal BPPV (PC-BPPV) has been shown in the previous studies [8].

Although CRM has a high success rate in BPPV treatment, sometimes BPPV show recurrence [9]. The recurrence of BPPV is an important issue. It leads to common symptoms of BPPV, lack of patients' trust in the medical team and unnecessary evaluations. It has been reported that recurrent BPPV is not rare [10]. Studies have shown that most of the recurrences occur in the 6 to 12 months after the first attack [11]. Different studies have reported variety of recurrence rate for BPPV from $13.5 \%[12,13]$ to $20-30 \%$ and $50 \%$ [14].

Otolithic signals play an important role in the perception of gravity, uprightness, postural control and linear acceleration. In BPPV, otoconia are detached from the utricle and this may affect otolith function as well [15]. This detachment may be due to a degenerative process in otolith organs [6] that may lead to the high possibility of BPPV, its resistance to treatment and recurrence.

Recently, through relatively new tests of otolith function including cervical vestibular evoked myogenic potential (cVEMP) and ocular vestibular evoked myogenic potential (oVEMP), we are able to detect otolith dysfunctions. The cVEMP evaluates saccule and inferior vestibular nerve. The oVEMP is presumably originated from the utricle and superior vestibular nerve. It has been shown that utricular dysfunction and oVEMP abnormalities are highly correlated [16].

We assumed that otolith dysfunction might be a risk factor for BPPV recurrence. It is assumed that habituation rehabilitation might be beneficial in patients with BPPV who suffer from otolith dysfunction and might reduce BPPV recurrence rate. Therefore, the aims of the present study were: 1) to examine the effects of otolith dysfunction on recurrence of BPPV and 2) to describe the effect of a habituation rehabilitation program on BPPV recurrence in BPPV patients with otolith dysfunction.

\section{Subjects and Methods}

This clinical trial study was conducted on 45 age-matched patients aged 20-60 years-old, with PC-BPPV in the DixHallpike test (upbeating and torsional nystagmus). Patients with BPPV were then divided into three groups: 1) The first group included subjects with normal cVEMP and oVEMP in both ears and they received a CRM maneuver. 2) The second group included subjects with abnormal cVEMP and/or oVEMP in the PC-BPPV side and they received a CRM maneuver. 3) The third group had abnormal cVEMP and/or oVEMP in the PC-BPPV side and they received CRM maneuver and two months of habituation and otolith rehabilitation. The time interval between the onset of BPPV and the treatment were less than two weeks.

Meniere's disease, endolymphatic hydrops, labyrinthitis, vestibular neuritis, cervical problems, trauma history, migraine, otologic or non-otologic surgery including maxillofacial and dental surgery, sudden sensory neural hearing loss, conductive hearing loss, prolonged bed rest and patient refusal to participate in the study were exclusion criteria. Patients underwent following tests to check exclusion criteria: neurologic examination, history taking, immittance acoustic (Zodiac 901, Madsen, GN Otometrics, Denmark), audiometry (AC40, Interacoustic, Assens, Denmark), EcochG with TipTrode (ICS Chartr EP, GN Otometrics, Taastrup, Denmark), Bithermal Caloric test (VNG Eye Dynamics Inc., Micromedical, Chatham, IL, USA).

The cVEMP recordings were performed using ICS Chartr EP manufactured by BioLogic (GN Otometrics) with $500 \mathrm{~Hz}$ tone burst at $95 \mathrm{~dB}$ normalized hearing level (nHL), with alternating polarity, band pass filter $10-1,500 \mathrm{~Hz}, 5.1 / \mathrm{s}$ rate with $50 \mathrm{~ms}$ time window. Sweep number was 150 . Stimuli were delivered through TDH39 headphones (Telephonics, Farmingdale, NY, USA). The electrode array was as follows: the reference electrode on a middle part of the sternocleidomastoid muscle, the active electrode on an upper part of the sternum, the ground electrode on the forehead. Subjects turned their head to the contralateral side while sitting in the chair. The device showed and monitored muscle contraction (RMS) automatically. The amplitude of biphasic p13-n23 wave was measured from the most positive peak of the wave (p13) to the most negative peak (n23) in microvolts.

The oVEMP recordings were conducted in the sitting position while the patient was looking upward at a small target that was within $100 \mathrm{~cm}$. The visual angle was about 30 degree as this angle has been shown to produce the best response in comparison to other eye positions [17]. The electrode array was as follows: the active electrode on the face approximately $1 \mathrm{~cm}$ below the contralateral lower eyelid, the reference electrode on the chin $1 \mathrm{~cm}$ below the active electrode, and the ground electrode on the forehead. Using ICS Chartr EP manufactured by BioLogic (GN Otometrics), oVEMP was recorded by $500 \mathrm{~Hz}$ tone bursts at $95 \mathrm{~dB} \mathrm{nHL}$ with alternating polarity, the bandpass filter of $10-1,500 \mathrm{~Hz}$ and $5.1 / \mathrm{s}$ rate. 
The analysis window was $75 \mathrm{~ms}$ and responses to 200 stimuli were averaged. The initial negative-positive biphasic waveform comprised peaks N1 and P1. We analyzed the peak-topeak amplitude of N1 and P1. Otolith dysfunction was defined as the lack of unilateral responses in cVEMP and oVEMP.

Epley's maneuver was performed on all subjects with BPPV. One week after treatment they were assessed with Dix-Hallpike test and if there was no nystagmus and/or vertigo, treatment was considered successful. If symptoms were persistent, Epley's maneuver was repeated for the second time. For subjects in group 3, a rehabilitation program including habituation and otolith exercises were utilized. Exercises included: jumping on the trampoline-like surface with eyes open and closed, walking on the inflated surface with eyes open and closed (head straight and head tilted in different directions), reading a text with linear head movement, standing on the tilt board and using an exercise ball. The duration of the rehabilitation program was two months and subjects were evaluated weekly to modify the exercises based on their performance. Follow-up care included communication by phone and in case of symptoms recurrence, repetition of the Epley's maneuver was done.

The study was approved by ethical committee of Tehran University of Medical Sciences with number 260-316. Informed consent was obtained from all participants.

For statistical analysis, SPSS 19.0 software (IBM Corp., Armonk, NY, USA) was used. The significance level was considered 0.05. Data were analyzed using Pearson correlation and chi-square test.

\section{Results}

45 patients from 22 to 60 years old were included. The

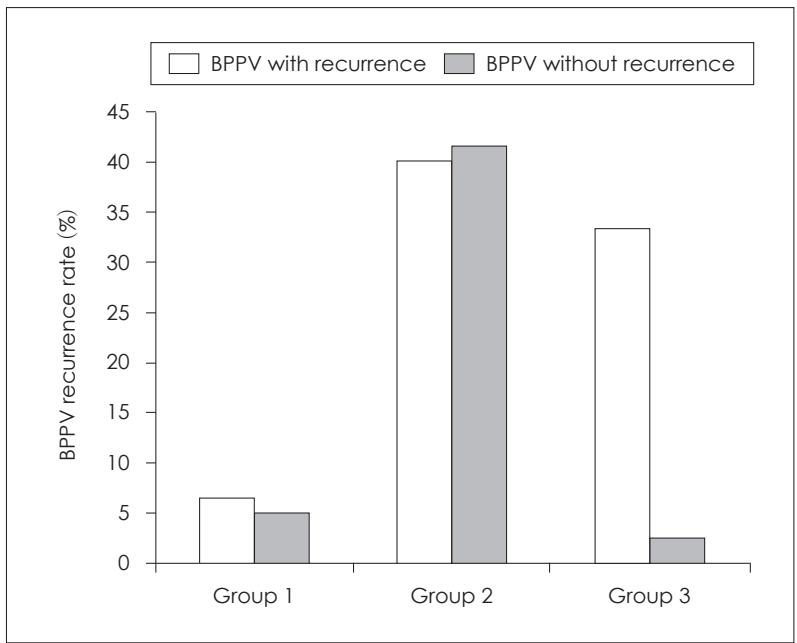

Fig. 1. BPPV recurrence rate in three groups before and after rehabilitation. BPPV: benign paroxysmal positional vertigo. mean age of patients in the group without otolith dysfunction was $45.46 \pm 7.39$, in the group 2 was $44.80 \pm 9.70$ and in the group 3 was $42.40 \pm 10.04$. There were 6 males and 9 females in group 1, 8 males and 7 females in group 2, and 5 males and 10 females in groups 3 . The rate of BPPV recurrence before rehabilitation was $6.7 \%, 40 \%$, and $33.3 \%$ in group 1,2 , and 3 , respectively. After rehabilitation, the rate of BPPV recurrence was $5.2 \%, 41.5 \%$, and $2.7 \%$ in group 1,2 , and 3 respectively. Fig. 1 shows BPPV recurrence rate in 3 groups before and after rehabilitation.

In Table 1, the number and percent of BPPV recurrence in each group are shown. BPPV recurrence is more prevalent in groups 2 and 3. Comparison of BPPV recurrence among groups with chi-square test is shown in Table 2. The difference between groups 1 and 2 was statistically significant. The BPPV recurrence in groups 2 and 3 in patients with cVEMP and/or oVEMP abnormalities is shown in Table 3 and 4. Comparison of otolith abnormalities and BPPV recurrence in groups 2 and 3 with chi-square test is shown in Table 5 . It shows that in both groups BPPV recurrence was significantly correlated with otolith dysfunction.

Comparison of BPPV recurrence among groups was performed by chi-square $\left(\chi^{2}\right)$ test. The difference between groups 1 and 2 and also between group 1 and 3 were statistically significant $(p<0.05)$. In general, $5 \%$ of patients with a cVEMP abnormality, $12 \%$ of patients with oVEMP abnormality, and

Table 1. Number and percent of benign paroxysmal positional vertigo recurrence in each group

\begin{tabular}{cccc}
\hline & $\begin{array}{c}\text { Recurrent } \\
\text { (percent) }\end{array}$ & $\begin{array}{c}\text { Not recurrent } \\
\text { (percent) }\end{array}$ & $\begin{array}{c}\text { Total } \\
\text { (percent) }\end{array}$ \\
\hline Group 1 & $1(6.7)$ & $14(93.3)$ & $15(100)$ \\
Group 2 & $6(40)$ & $9(60)$ & $15(100)$ \\
Group 3 & $2(13.3)$ & $13(86.7)$ & $15(100)$ \\
\hline Total & $9(20)$ & $36(80)$ & $45(100)$ \\
\hline
\end{tabular}

Table 2. Comparison of benign paroxysmal positional vertigo recurrence among groups

\begin{tabular}{lccc}
\hline & Value & df & p value \\
\hline Groups 1 and 2 & 4.658 & 1 & 0.031 \\
Groups 1 and 3 & 0.370 & 1 & 0.543 \\
Groups 2 and 3 & 2.727 & 1 & 0.099 \\
\hline
\end{tabular}

Table 3. Recurrence of benign paroxysmal positional vertigo in group 2 in patients with cVEMP and/or oVEMP abnormalities

\begin{tabular}{cccc}
\hline & Recurrent & Not recurrent & Total \\
\hline cVEMP abnormality & 2 & 8 & 10 \\
OVEMP abnormality & 4 & 1 & 5 \\
\hline Total & 6 & 9 & 15 \\
\hline
\end{tabular}

cVEMP: cervical vestibular evoked myogenic potential, oVEMP: ocular vestibular evoked myogenic potential 
Table 4. Recurrence of benign paroxysmal positional vertigo in group 3 in patients with cVEMP and/or oVEMP abnormalities

\begin{tabular}{lccc}
\hline & Recurrent & $\begin{array}{c}\text { Not } \\
\text { recurrent }\end{array}$ & Total \\
\hline cVEMP abnormality & 0 & 10 & 10 \\
OVEMP abnormality & 2 & 2 & 4 \\
cVEMP and oVEMP abnormality & 0 & 1 & 1 \\
\hline \multicolumn{1}{c}{ Total } & 2 & 13 & 15 \\
\hline
\end{tabular}

cVEMP: cervical vestibular evoked myogenic potential, oVEMP: ocular vestibular evoked myogenic potential

Table 5. Correlation of otolith abnormalities with benign paroxysmal positional vertigo recurrence

\begin{tabular}{cccc}
\hline & Value & df & p value \\
\hline Group 2 & 5.00 & 1 & 0.025 \\
Group 3 & 6.34 & 2 & 0.042 \\
\hline
\end{tabular}

$5 \%$ of patients with both cVEMP and oVEMP abnormalities showed recurrent BPPV (group 2 and 3). Therefore $73.3 \%$ of patients with cVEMP and/or oVEMP abnormalities in group 2 and 3 showed recurrent BPPV. Spearman correlation analysis showed a significant correlation between recurrent BPPV and otolithic abnormalities $(\mathrm{r}=0.81 ; p<0.05)$.

Chi-square $\left(\chi^{2}\right)$ test showed a significant decrease in BPPV recurrence rate in group 3 after repositioning rehabilitation and otolithic exercise $(p<0.05)$. On the other hand, chi-square $\left(\chi^{2}\right)$ test showed no significant decrease in BPPV recurrence rate in group 2 following only repositioning maneuver ( $p>$ $0.05)$.

\section{Discussion}

This study was conducted on 45 subjects with BPPV. In groups 1,2 , and 3 , recurrence rate were $6.7 \%, 40 \%$, and $13.3 \%$, respectively. Other studies have reported the BPPV recurrence rate from $13.5 \%$ to $50 \%[12,13,18]$. Variability in reported BPPV recurrence may be related to patient variables such as age, sample size, and treatment maneuver. This study showed that patients without dysfunction of otolith organs had less BPPV recurrence. In group 2, we found more BPPV recurrence in comparison to groups 1 and 3 and there was a significant correlation between BPPV recurrence and otolith dysfunction (oVEMP abnormality). This shows the possible effects of otolith dysfunction on BPPV recurrence. Degenerated saccular or utricular structures might lead to more BPPV recurrence. A consistent detachment of otoconia particles that move to posterior semicircular canal might cause BPPV [6,19]. Evaluating otolith function may have a potential use for determining patients' prognosis about BPPV treatments and probable BPPV recurrence. This may help clinician counsel the patient regarding follow-up evaluations and treatments. It has been shown that endolymphatic hydrops is a risk factor for BPPV recurrence [20] and the present study showed that otolith dysfunction may be another important risk factor.

The BPPV recurrence was seen more prevalent in patients with oVEMP abnormalities which represents utricle involvements. Patients with cVEMP abnormalities showed no significant difference regarding BPPV recurrence rate in comparison with group 1. cVEMP can evaluate saccular function. It is supposed that otoconia particles inducing BPPV are generally detached from utricle which is in close anatomic proximity to the semicircular canal $[1,21]$.

Despite the fact that Epley's maneuver is an effective, safe and the simple treatment for BPPV, to date, treatment of BPPV patients has been limited to this maneuver and other similar CRM maneuvers. The present study showed that a proportion of patients with BPPV suffer from otolith dysfunction. In this population, adding habituation and otolith rehabilitation exercises to Epley's maneuver can significantly decrease BPPV recurrence rate. The rate of BPPV recurrence was $13.3 \%$ and $40 \%$ in group 2 and 3 , respectively. Other studies have shown that habituation exercises are beneficial for BPPV patients. These daily exercises may prevent the accumulation of otoconia in the posterior canal by dispersing otoconia particles in patients with recurrent BPPV, so BPPV recurrence will be decreased in extended time.

In conclusion, this study showed that the BPPV recurrence rate was higher in patients with otolith dysfunction, especially utricular dysfunction, and habituation and otolith exercises in addition to traditional CRM maneuvers can decrease BPPV recurrence rate significantly. Otolith dysfunction may be a good predictor of BPPV recurrence and may help with consulting the patient and arranging the follow-up sessions and planning the rehabilitation program.

\section{Acknowledgments}

We thank from Dr. Pedram Borghei who provided insight and expertise that greatly assisted the research. This study was a part of the first author Ph.D. thesis entitled "The effect of vestibular rehabilitation on BPPV recurrence and degeneration of otolithic organs in idiopathic BPPV patients" sponsored by Tehran University of medical sciences, school of rehabilitation, audiology department, at 2013.

\section{Conflicts of interest}

The authors have no financial conflicts of interest.

\section{REFERENCES}

1) Hornibrook J. Benign paroxysmal positional vertigo (BPPV): history, pathophysiology, office treatment and future directions. Int J Otolaryngol 2011;2011:835671.

2) Pollak L, Kushnir M, Goldberg HS. Physical inactivity as a contributing factor for onset of idiopathic benign paroxysmal position- 
al vertigo. Acta Otolaryngol 2011;131:624-7.

3) Korres S, Gkoritsa E, Giannakakou-Razelou D, Yiotakis I, Riga M, Nikolpoulos TP. Vestibular evoked myogenic potentials in patients with BPPV. Med Sci Monit 2011;17:CR42-7.

4) Mandalà M, Santoro GP, Awrey J, Nuti D. Vestibular neuritis: recurrence and incidence of secondary benign paroxysmal positional vertigo. Acta Otolaryngol 2010;130:565-7.

5) Dundar MA, Derin S, Aricigil M, Eryilmaz MA, Arbag H. Posttraumatic refractory multiple canal benign paroxysmal positional vertigo: a case report. North Clin Istanb 2016;3:229-32.

6) von Brevern M, Bertholon P, Brandt T, Fife T, Imai T, Nuti D, et al. Benign paroxysmal positional vertigo: diagnostic criteria. J Vestib Res 2015;25:105-17.

7) Do YK, Kim J, Park CY, Chung MH, Moon IS, Yang HS. The effect of early canalith repositioning on benign paroxysmal positional vertigo on recurrence. Clin Exp Otorhinolaryngol 2011;4:113-7.

8) Pereira AB, Santos JN, Volpe FM. Effect of Epley's maneuver on the quality of life of paroxismal positional benign vertigo patients. Braz J Otorhinolaryngol 2010;76:704-8.

9) Korres S, Balatsouras DG, Kaberos A, Economou C, Kandiloros D, Ferekidis E. Occurrence of semicircular canal involvement in benign paroxysmal positional vertigo. Otol Neurotol 2002;23:926-32.

10) Choi SJ, Lee JB, Lim HJ, Park HY, Park K, In SM, et al. Clinical features of recurrent or persistent benign paroxysmal positional vertigo. Otolaryngol Head Neck Surg 2012;147:919-24.

11) Steenerson RL, Cronin GW, Marbach PM. Effectiveness of treatment techniques in 923 cases of benign paroxysmal positional vertigo. Laryngoscope 2005;115:226-31.

12) Macias JD, Lambert KM, Massingale S, Ellensohn A, Fritz JA. Variables affecting treatment in benign paroxysmal positional ver- tigo. Laryngoscope 2000;110:1921-4.

13) Sridhar $S$, Panda N. Particle repositioning manoeuvre in benign paroxysmal positional vertigo: is it really safe? J Otolaryngol 2005;34: 41-5.

14) Pérez P, Franco V, Cuesta P, Aldama P, Alvarez MJ, Méndez JC. Recurrence of benign paroxysmal positional vertigo. Otol Neurotol 2012;33:437-43

15) Ernst A, Basta D, Seidl RO, Todt I, Scherer H, Clarke A. Management of posttraumatic vertigo. Otolaryngol Head Neck Surg 2005; 132:554-8.

16) Jacobson GP, McCaslin DL, Piker EG, Gruenwald J, Grantham SL, Tegel L. Patterns of abnormality in cVEMP, oVEMP, and caloric tests may provide topological information about vestibular impairment. J Am Acad Audiol 2011;22:601-11.

17) Chihara Y, Iwasaki S, Ushio M, Murofushi T. Vestibular-evoked extraocular potentials by air-conducted sound: another clinical test for vestibular function. Clin Neurophysiol 2007;118:2745-51.

18) Brandt T, Huppert D, Hecht J, Karch C, Strupp M. Benign paroxysmal positioning vertigo: a long-term follow-up (6-17 years) of 125 patients. Acta Otolaryngol 2006;126:160-3.

19) von Brevern M, Schmidt T, Schönfeld U, Lempert T, Clarke AH. Utricular dysfunction in patients with benign paroxysmal positional vertigo. Otol Neurotol 2006;27:92-6.

20) Tanimoto H, Doi K, Nishikawa T, Nibu K. Risk factors for recurrence of benign paroxysmal positional vertigo. J Otolaryngol Head Neck Surg 2008;37:832-5.

21) Inagaki T, Suzuki M, Otsuka K, Kitajima N, Furuya M, Ogawa $Y$, et al. Model experiments of BPPV using isolated utricle and posterior semicircular canal. Auris Nasus Larynx 2006;33:129-34. 